\title{
The liquid penetration of diesel substitutes
}

\author{
Sebastian Riess ${ }^{1,2}$, Lukas Weiss ${ }^{* 1,2}$, Javad Rezaei ${ }^{1}$, Andreas Peter ${ }^{1}$, Michael Wensing ${ }^{1,2}$ \\ ${ }^{1}$ Institute of Engineering Thermodynamics (LTT), FAU Erlangen-Nürnberg, Germany \\ ${ }^{2}$ Erlangen Graduate School in Advanced Optical Technologies (SAOT), \\ FAU Erlangen-Nürnberg, Germany \\ *Corresponding author: lukas.weiss@fau.de
}

\begin{abstract}
Diesel fuel consist of several hundreds of substances on organic basis. Experimental and numerical investigations of this multicomponent fuel are hard to interpret in detail, since the behavior of the multicomponent mixture is complex. Physical and chemical data of this system is not available under engine relevant conditions. Instead, fundamental research substitutes diesel with pure substances, where a big database exists.

Prior work already showed, that overall spray propagation (including vapor phase) is nearly independent on the injected fuel. This is due to the high air entrainment at present diesel engine conditions (very high injection pressure and dense ambient atmosphere). The high air entrainment shortly behind the nozzle exit (within the first $5 \mathrm{~mm}$ penetration) creates a situation where properties of the ambient gas dominate the spray propagation resulting in similar mass and momentum distributions even for different fuels, if the injection conditions are kept constant. On the other hand, the liquid length is clearly different for different fuels, so that location and time of the phase change differ with consequences on the time available for mixture formation in the gas phase. The paper describes the liquid length as a function of the enthalpy necessary for the phase transition (given by the fuel and fuel temperature at injection) and the injection conditions (ambient gas properties, injector design and injection pressure). We compare two different models describing the enthalpy balance. Siebers et al. presented "Model I", where mass transfer dominates the enthalpy transfer and evaporation takes place. In our own "Model II" evaporation is suppressed, resulting in a heat transfer driven enthalpy transfer without mass transport. The calculations are validated with experimental data.

The liquid length is optically accessible by Mie-Scattering imaging techniques, the complete spray evolution by Schlieren technique. The experimental study was carried out in the high-pressure combustion vessel "OptiVeP" at FAU. The data shown in this paper derived from measurements with dodecane injected at 1200 bar into $613 \mathrm{~K}$ ambient. The ambient pressure varies from 1-10 MPa. A Continental research injector with a $115 \mu \mathrm{m}$ hole and L/D of 6.5 was used. Nitrogen atmosphere suppressed ignition.

Increasing the ambient pressure leads to a change in the mechanism in phase transition. It switches from a mass transfer dominated regime to a heat transfer dominated regime at high ambient pressures.
\end{abstract}

\section{Keywords}

Diesel, liquid-penetration, enthalpy

\section{Introduction}

Diesel injection is in focus of research for many years now, but still basic parameters like the liquid length are not physically described yet. One problem is that no fluid data of multicomponent systems under relevant ambient conditions are available. Thus, research often substitutes diesel with pure substances like dodecane.

Dennis Siebers delivered the most comprehensive description of diesel fuel injection in the late 90 s. He summarized his findings in his scaling law [2], which will be reviewed later on. During the injection a momentum transfer from fuel to entrained air occurs. The spray velocity decreases with increasing mass of entrained air. The amount of entrained air is very high. Yue et al. showed with $\mathrm{x}$-ray absorption, that the fuel volumetric fraction on the spray axis decreases to 0.8 at $1 \mathrm{~mm}$ distance to the nozzle and to 0.2 at $6 \mathrm{~mm}$ to the nozzle [3]. Leick et al. confirmed the results with a similar technique under modern diesel relevant ambient conditions $\left(\rho_{a}=21.7 \mathrm{~kg} / \mathrm{m}^{3} ; p_{i n j}=135 \mathrm{MPa}\right)$ [4]. Riess et al. extended this view and showed that the extreme high air entrainment is fuel independent. They investigated different Diesel-Ethanol blends with Raman spectroscopy, where local substance amount ratios can be determined. They found similar high air entrainment ratios and additionally showed that mixture formation is independent from the fuel, which results in similar penetration curves for all blends and pure substances in Schlieren measurements. The maximum liquid penetration from the orifice, though is strongly determined by the fuel [5]. This maximum liquid length is called "liquid length" further on in this paper, see Figure 2. 


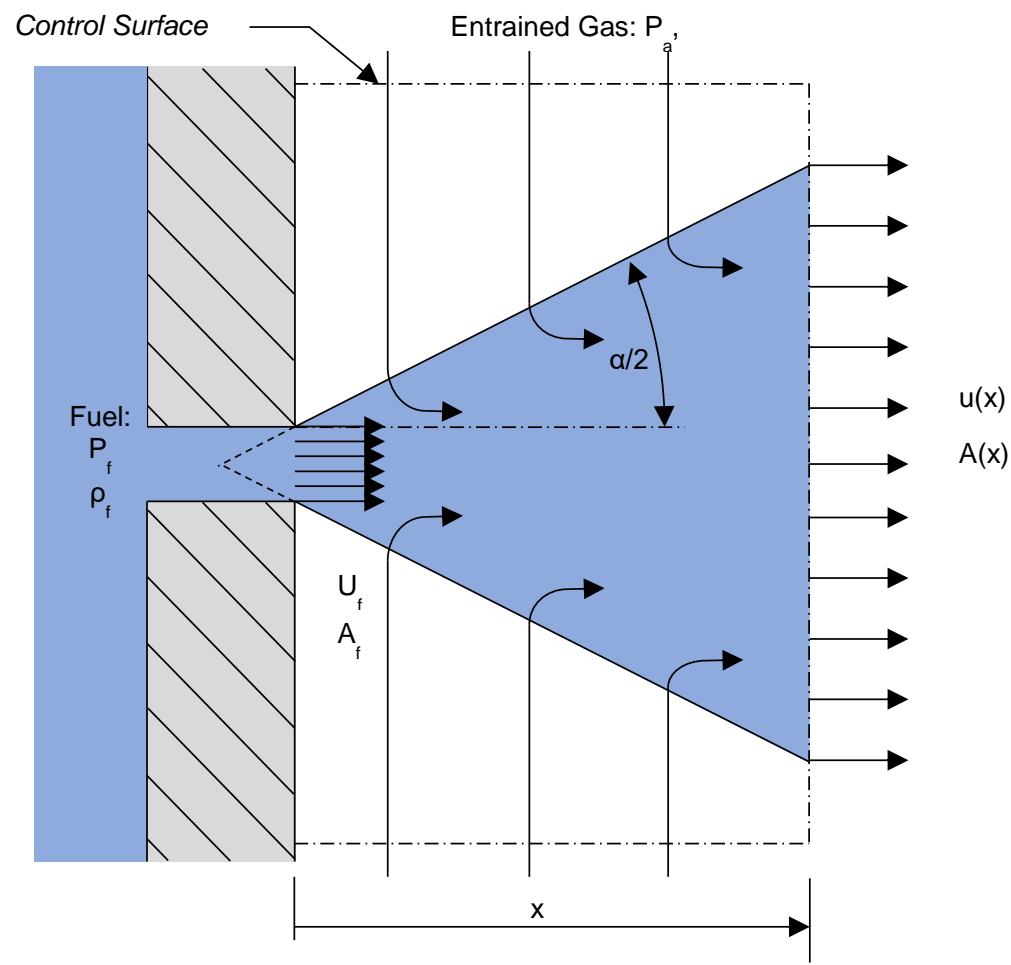

Figure 1. Schematic of the spray model according to Siebers [1]

\section{Volume and momentum balance}

Naber and Siebers formulated a momentum model for a full diesel spray under non-vaporizing conditions, that explains the fuel independency from Riess et al. [1,5]. His schematic view is drawn in Figure 1. The momentum source is the fuel injection with mass rate $\dot{m}_{f}$ and exit velocity $u_{0}$. The momentum is transferred to the entrained air $\dot{m}_{a}$. The spray velocity $u(x)$ is decreasing with distance to the orifice and thus with increasing entrained air mass.

$\dot{m}_{f} \cdot u_{0}=\left(\dot{m}_{f}+\dot{m}_{a}\right) \cdot u(x)=$ const.

Since the fuel volume in the spray is negligible small, the spray cross section that is covered by fuel $A_{f}$ is also negligible [5]. Thus the complete spray cross section can be assigned to the entrained air and the resulting air mass flow can be estimated from the momentum balance (1) and the continuum constraints (2) and (3).

$$
\begin{aligned}
& \dot{m}_{f}=\rho_{f 0} \cdot A_{0} \cdot u_{0} \\
& \dot{m}_{a}=\rho_{a} \cdot A(x) \cdot u(x)
\end{aligned}
$$

In this model several assumptions were made:

1) $u(x)$ is constant in every cross section (rectangular velocity profile in the spray)

2) air only moves in radial direction outside the spray [6]

3) orifice exit velocity $u_{0}$, spray spreading angle $\alpha$ and nozzle discharge coefficient $C_{a}$ are constant [5]

4) no-slip condition between air and fuel

5) constant ambient density $\rho_{a}$

Siebers et al. defined a dimensionless orifice distance $\tilde{x}$ for better comparability between different ambient conditions (4). In addition, they defined the mass flow ratio $B$ as function of this dimensionless distance (5).

$$
\begin{aligned}
& \tilde{x}=\sqrt{\frac{\rho_{a}}{\rho_{f}}} \cdot \frac{x}{\sqrt{C_{a}} \cdot d} \cdot a \cdot \tan \left(\frac{\alpha}{2}\right) \\
& \frac{\dot{m}_{f}}{\dot{m}_{a}}:=B=\frac{2}{\sqrt{1+16 \tilde{x}}-1}
\end{aligned}
$$


With equation (4) the non-vaporizing spray penetration is described and equation (5) gives the according mass ratios. The equations also show, that the mass ratio in a certain distance $\mathrm{x}$ is independent from the injection pressure. Consequently an increase in injection pressure does not increase the air entrainment. Schlieren measurements from Riess et al. confirm the proposed model even for the full spray with vaporizing conditions [5]. The gaseous spray penetrates steadily independent from the fuels physical state, which again shows the dominating role of the entrained air.

\section{Calculation of the liquid length}

The liquid length $L$ of a diesel spray is the maximum penetration of liquid fuel. Under vaporizing conditions, the liquid length is constant as long as the fuel mass flow is constant, see Figure 2. At this distance, enough ambient gas is mixed in and thus enthalpy is transported into the spray to vaporize all fuel. According to equation (5) this distance is assigned to a certain mass ratio. Two simplified models are considered:

Calculation model l: Mass transport is much faster than heat transfer $\rightarrow$ Fuel droplets evaporate and mass transport is only limited by the saturation of the ambient. The mixture temperature $T_{m i x}$ is a consequence of the evaporation cooling and heating due to the entrained hot ambient gas. (Siebers scaling law)

Calculation model II: Heat transfer is much faster than mass transport $\rightarrow$ Fuel droplets do not evaporate at all until they have reached their phase change temperature (boiling or critical). Enthalpy is provided by entrained hot air.

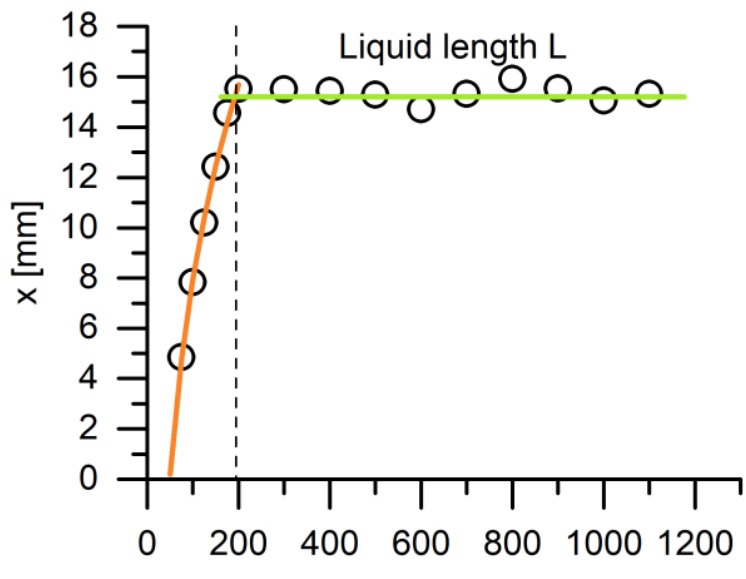

$\mathrm{t}[\mu \mathrm{s}]$

Figure 2. Definition of the liquid length

\section{Calculation model I}

The idea of Siebers' scaling law is to search for the mass ratio at which the enthalpy balance and the resulting partial pressure ratios are satisfied. The general enthalpy balance for an evaporating spray is:

$$
\dot{m}_{a}\left(h_{a}\left(T_{a}, p_{a}\right)-h_{a}\left(T_{a 2}, p_{a 2}\right)\right)=\dot{m}_{f}\left(h_{f}\left(T_{f 2}, p_{f 2}\right)-h_{f}\left(T_{f}, p_{a}\right)\right)
$$

with the thermodynamic equilibrium at:

$$
T_{a 2}=T_{f 2}=T_{m i x}
$$

The equilibrium pressures are equal to the partial pressures $\tilde{p}_{i}$ satisfying Dalton's law (9):

$$
\begin{aligned}
& p_{a}=p_{a 2}+p_{f 2}=\tilde{p}_{a}+\tilde{p}_{f} \\
& \tilde{p}_{i}=\dot{m}_{i} \cdot \frac{z_{i}}{M_{i}} \cdot \frac{R_{m} \cdot T_{m i x}}{\dot{V}}
\end{aligned}
$$


The scaling law assumes a much faster mass transport than heat transfer, which means, that the environment is always saturated of fuel vapor. Thus, the partial pressure of the fuel is equal to the saturation pressure $p_{s}(T)$ at a certain condition:

$$
\begin{aligned}
& \tilde{p}_{f}=p_{s}\left(T_{\text {mix }}\right) \\
& \tilde{p}_{a}=p_{a}-p_{s}\left(T_{\text {mix }}\right)
\end{aligned}
$$

To allow a complete phase change, the enthalpy and the partial pressure balances have to result in the same value for the mass ratio at a certain mixture temperature $T_{\text {mix }}$ and have to be solved iteratively:

$$
\frac{1}{B_{L}}=\frac{\dot{m}_{a}}{\dot{m}_{f}}=\frac{h_{f}\left(T_{m i x}, p_{s}\left(T_{m i x}\right)\right)-h_{f}\left(T_{f}, p_{a}\right)}{h_{a}\left(T_{a}, p_{a}\right)-h_{a}\left(T_{m i x},\left(p_{a}-p_{s}\left(T_{m i x}\right)\right)\right)}=\frac{\left(p_{a}-p_{s}\left(T_{m i x}\right)\right) \cdot M_{a} \cdot z_{f}}{p_{s}\left(T_{m i x}\right) \cdot M_{f} \cdot z_{a}}
$$

With the known mass ratio $\mathrm{B}$, equation (5) can be solved for the corresponding dimensionless distance $\tilde{x}\left(B_{L}\right)$, which is defined as the dimensionless liquid length $\tilde{L}$.

\section{Calculation model II}

The second model assumes heat transfer to be much faster than mass transport. Thus no mass transfer to in the vapor phase is taken into account during heating up of the fuel. Enthalpy is transferred from the ambient gas to the liquid fuel until its phase change temperature is reached. The same general enthalpy balance like before is valid, see equation (6), but the equilibrium conditions have changed:

$$
T_{a 2}=T_{f 2}=T_{\text {mix }}=T_{\text {Phase Change }}
$$

The phase change temperature can be the boiling temperature or the critical temperature. Table 1 shows some phase change conditions for dodecane.

Table 1. Phase change conditions for dodecane [7]

\begin{tabular}{|l|c|c|c|}
\hline Pphase change & $1 \mathrm{MPa}$ & $1,5 \mathrm{MPa}$ & $p_{\text {crit }}=1,82 \mathrm{MPa}$ \\
\hline $\mathrm{T}_{\text {phase change }}$ & $614,5 \mathrm{~K}$ & $643 \mathrm{~K}$ & $658,1 \mathrm{~K}$ \\
\hline
\end{tabular}

The necessary mass ration can directly be calculated from equation (6). The according liquid length $\tilde{L}$ is again the solution of equatzion (5) for $\tilde{x}\left(B=B_{L}\right)$.

$$
\frac{1}{B_{L}}=\frac{\dot{m}_{a}}{\dot{m}_{f}}=\frac{h_{f}\left(T_{\text {Phase Change }}, p_{a}\right)-h_{f}\left(T_{f}, p_{a}\right)}{h_{a}\left(T_{a}, p_{a}\right)-h_{a}\left(T_{\text {Phase Change }}, p_{a}\right)}
$$

\section{Experimental methods}

The liquid length is optically accessible by Mie-Scattering imaging techniques, the complete spray evolution by Schlieren technique. The experimental study was carried out in the high-pressure combustion vessel "OptiVeP" at FAU. For more details see Riess et al. [5]. The operation point was constant at $873 \mathrm{~K}$ ambient temperature, $120 \mathrm{MPa}$ injection pressure and $363 \mathrm{~K}$ fuel temperature in nitrogen atmosphere, whereas the ambient pressure was varied from $1 \mathrm{MPa}$ to $9 \mathrm{MPa}$. A Continental 3 hole research injector was used. The spray spreading angles are derived from Schlieren images. The acquired Mie and Schlieren images where processed with a self-developed MATLABß code SprayAnalysis.

\section{Results and discussion}

This section starts with basic predictions by the two models, further the predicted liquid length are compared to the dodecane measurements

\section{Model Prediction - Enthalpy Balance}

The fuel enthalpy developing for different ambient pressures for both calculation models is drawn in Figure 3. With dominating mass transport (calculation model I) drawn in Figure 3a the initial enthalpy of the fuel $h_{f 0}$ is almost constant with rising pressure, whereas the enthalpy needed for evaporation $\Delta h_{f}$ is increasing steadily. A dominating heat transfer (calculation model II) leads to a complete different behavior, see Figure $3 \mathrm{~b}$. Here the enthalpy for the phase change $\Delta h_{\text {liq }}$ increases to a maximum, the decrease flats out forming a peak before a plateau. This 
developing is a direct consequence from the shape of the phase boundary line in the H-S diagram, see Figure 4. Since the critical point is placed in the turning point of the phase boundary line, the phase change enthalpy has a maximum below the critical pressure. Simultaneously, the evaporation enthalpy $\Delta h_{V}$ drops to zero as the critical point is reached. Thus the peak is even emphasized in the overall enthalpy $\Delta h_{\text {ges }}$. A suppressed mass transport demands a higher phase change enthalpy, which is mainly due to the much higher resulting mixing temperatures. In terms of the model assumptions these two models define upper and lower limits for the needed phase change enthalpy.
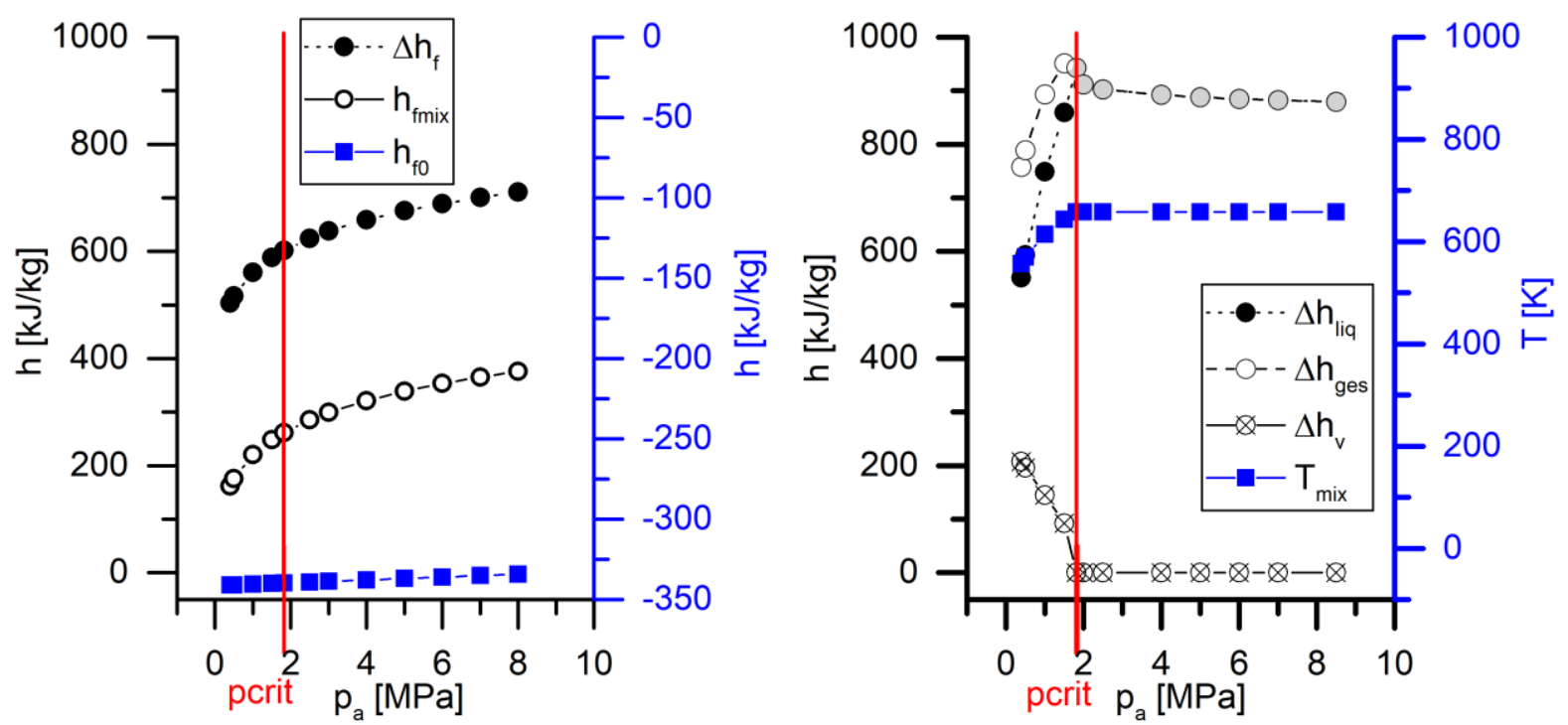

Figure 3. Enthalpy developing over pressure for calculation model I (a) and calculation model II (b)

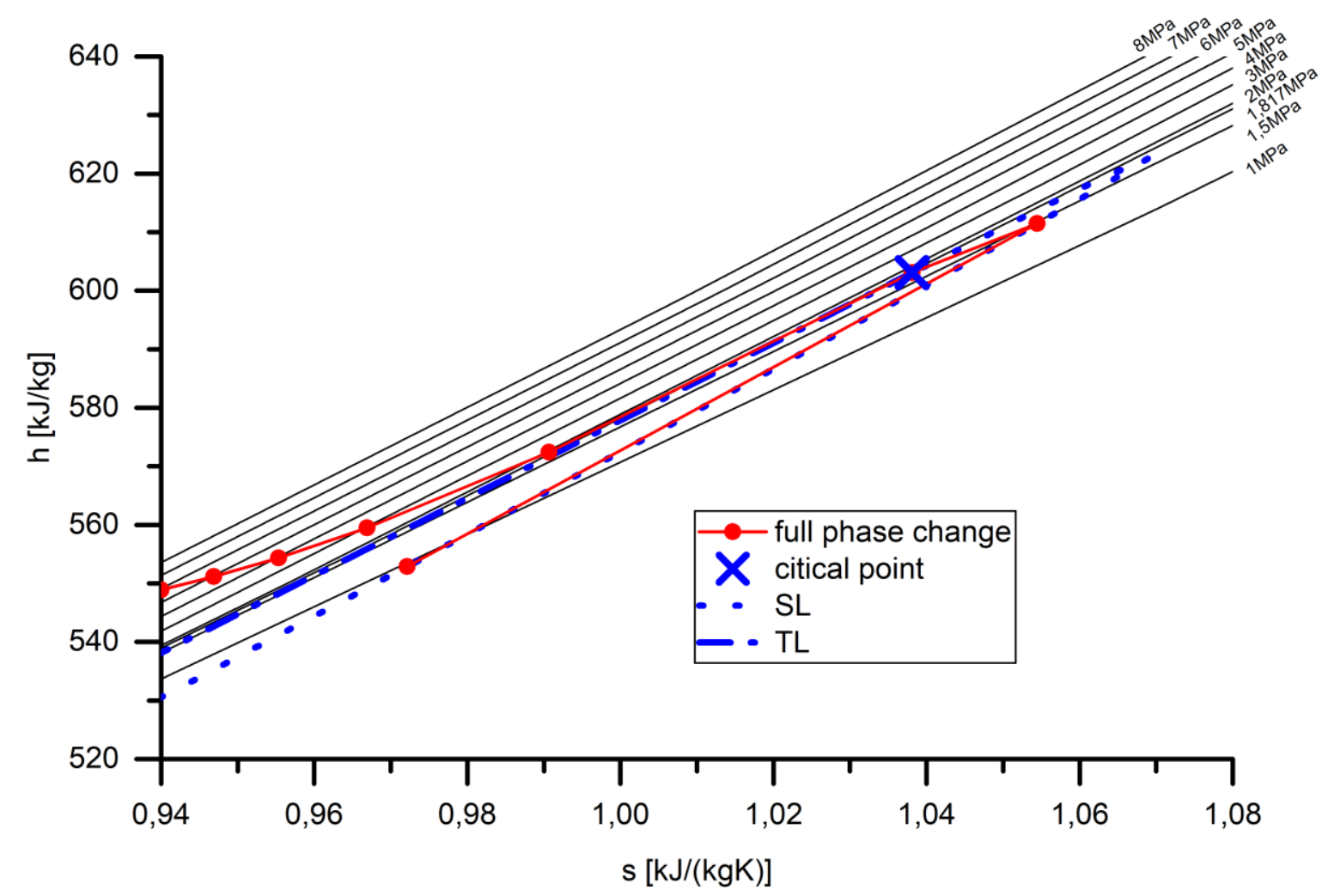

Figure 4. H-S-diagram for dodecane at $873 \mathrm{~K}$ with data from NIST [7] 
The peak in the enthalpy difference from calculation model II consequently results in a peak liquid length, see Figure 5. In contrast, model I predicts a steady decrease of the liquid length over pressure. It can also be seen, that model I underestimates and model II overestimates the liquid length, according to the upper and lower limits, depending on the mechanism of phase change. Siebers already noticed that and multiplied the calculated values with a suitable scaling factor. The best fits to the measured data points are shown in Figure 5. At a pressure of $3 \mathrm{MPa}$ a transition from model I to model II takes places. It even gets clearer in Figure 6, where the dimensionless liquid length is plotted. The peak in the dimensionless liquid length of model II cannot be reproduced experimentally. Instead, model I fits very well here. Above $3 \mathrm{MPa}$ ambient pressure model II predicts a constant dimensionless liquid length, which is confirmed by the experiment. Recalling the assumptions of the calculation models, at low ambient pressures mass transport dominates the liquid length. With increasing ambient pressure, this changes to a heat transfer dominated mechanism. The transition point seems to be fuel dependent, which calculations with different substances suggest. With these two models an experiment can be designed to confirm this conclusion. Measurements with other fuels and additional isothermal rows have to be carried out to clarify the transition between both mechanisms.

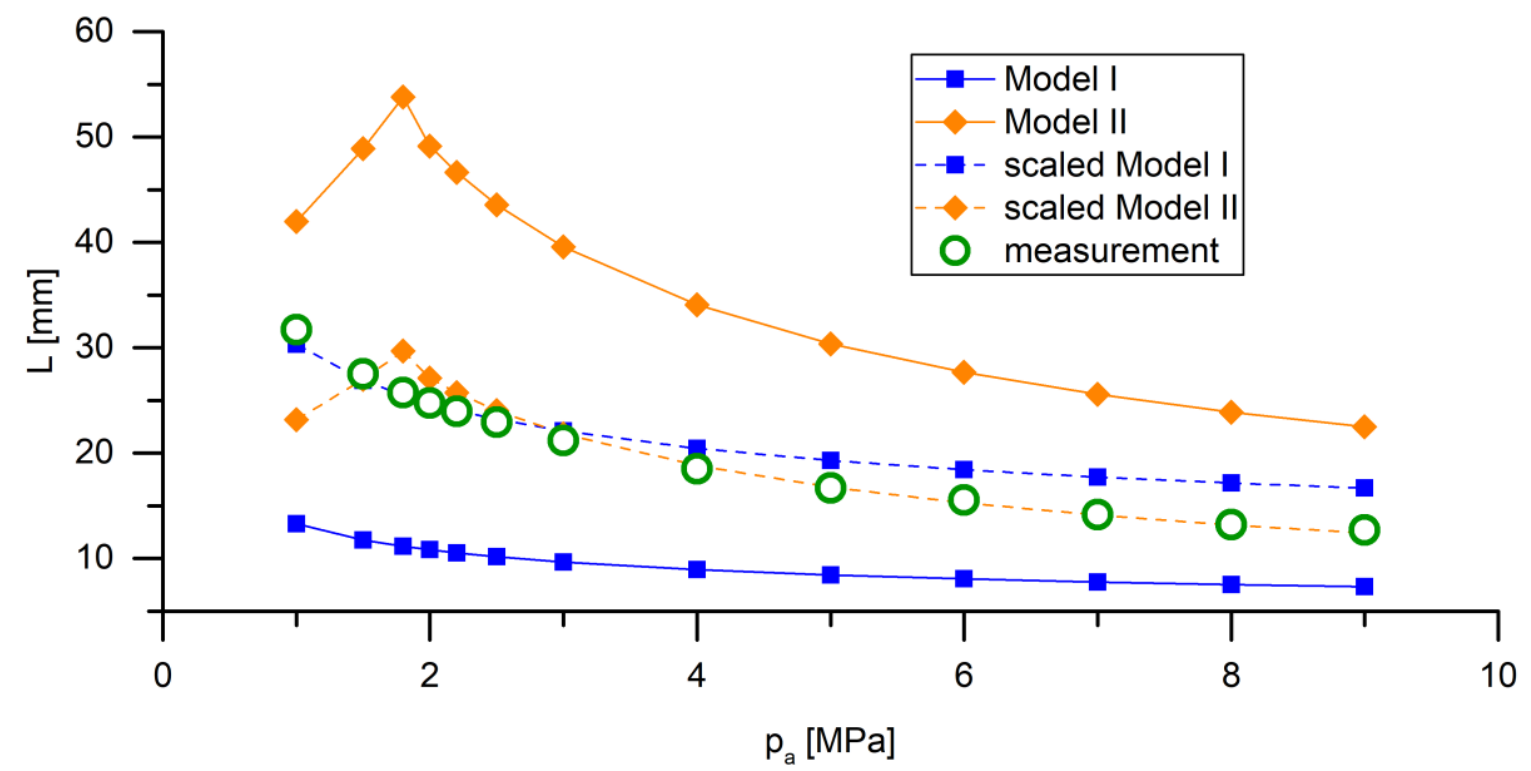

Figure 5. Comparison of the calculated liquid length according to model I and II with measured data of dodecane.

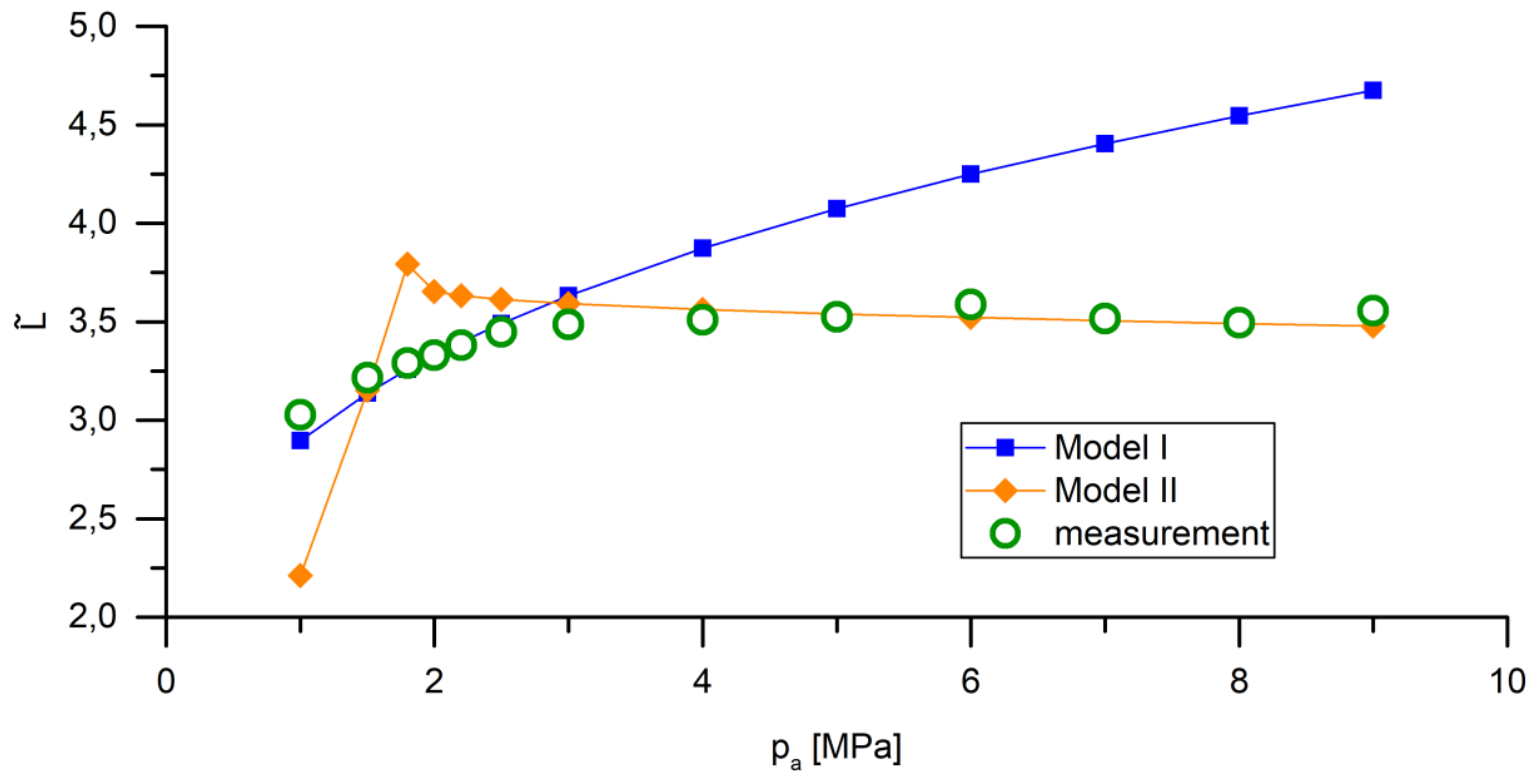

Figure 6. Fitted dimensionless liquid length to measured data of dodecane. 
One example of an additional isothermal row is presented in Figure 7. Dodecane was injected at $613 \mathrm{~K}$ ambient temperature. Model II fails to calculate any liquid length, because the temperature is below any phase change temperature at these pressures. Model I, which is expected to be predictive at low conditions, still promised a constant liquid length. This expectation could be confirmed in the experiment. A liquid length also occurs significantly below the boiling temperature, compare Table 1.

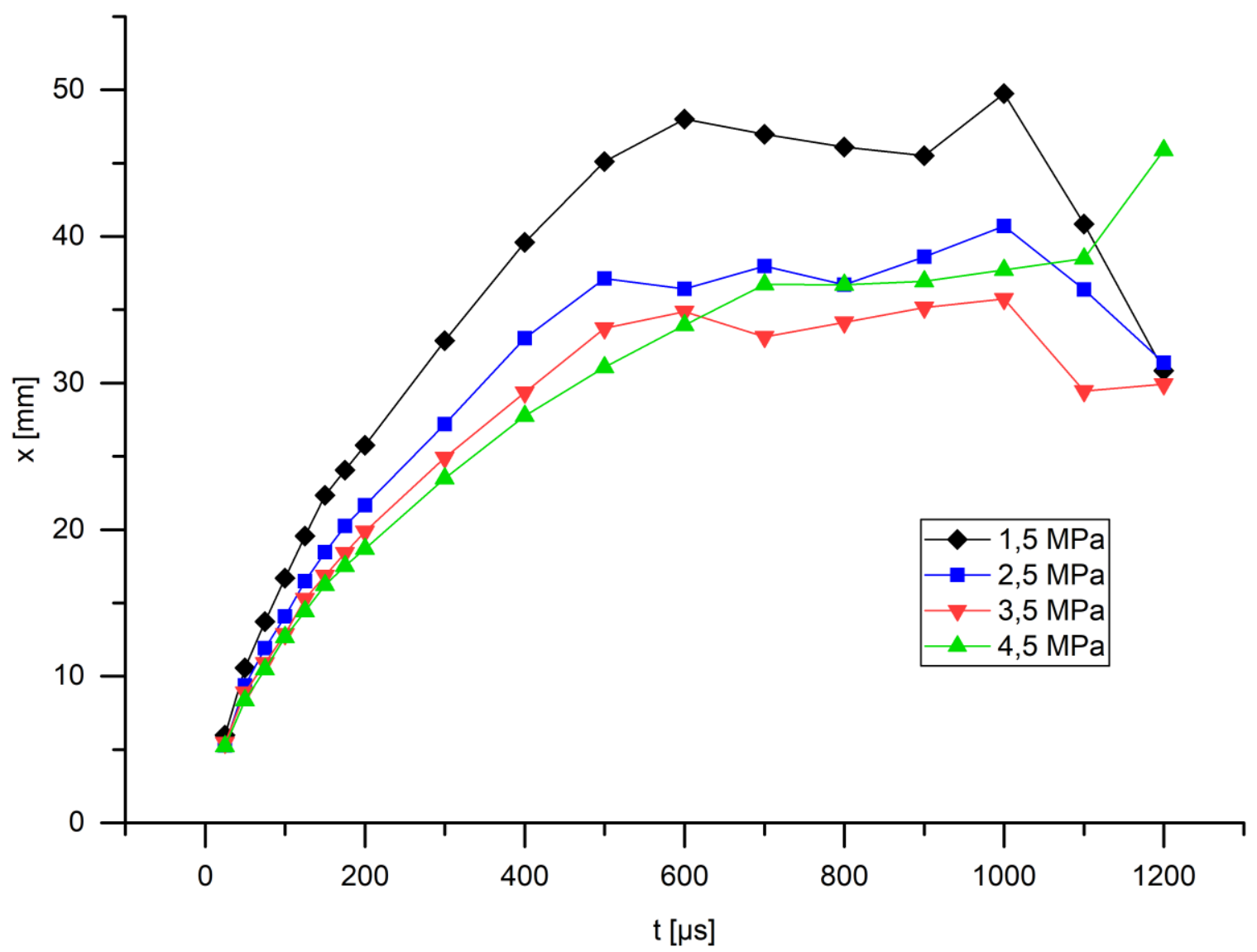

Figure 7. Liquid length of dodecane - pressure variation at $613 \mathrm{~K}$.

\section{Conclusions and outlook}

The full spray penetration under non-vaporizing and vaporizing conditions is described very well with Siebers' momentum balance and is confirmed by several authors. In contrast, the resulting liquid length is still not fully described, although a high effort is made in this research field. Siebers' scaling law, here called calculation model $I$, is a very good approach for low ambient pressures. The formation of a constant liquid length below boiling temperatures can be predicted qualitatively. The introduced calculation model II, in which the heat transfer is much faster than the mass transport, fits qualitatively very well at high ambient pressures. This concludes, that a change in the phase transition mechanism takes place from a mass transport dominated to a heat transfer dominated regime with increasing ambient pressure. The transition point seems to be fuel dependent. Similar experiments where already done with decane, hepatane, hexane, ethanol and rapeseedmethylester. Unfortunately only operation points at high ambient conditions with respect to the fluids phase transition temperatures and pressures where performed, thus a validation of the model results is not possible at that time.

\section{Nomenclature}

$\theta \quad$ spray angle $\left[{ }^{\circ}\right]$

$\rho_{a} \quad$ gas density $\left[\mathrm{kg} / \mathrm{m}^{3}\right]$

$\rho_{f 0} \quad$ fuel density (initial) $\left.\left[\mathrm{kg} / \mathrm{m}^{3}\right]\right]$

$\mathrm{P}_{\text {inj }} \quad$ injection pressure [MPa]

$\dot{m}_{f} \quad$ fuel mass flow [g/s]

$\dot{m}_{a} \quad$ air mass flow $[\mathrm{g} / \mathrm{s}]$ 


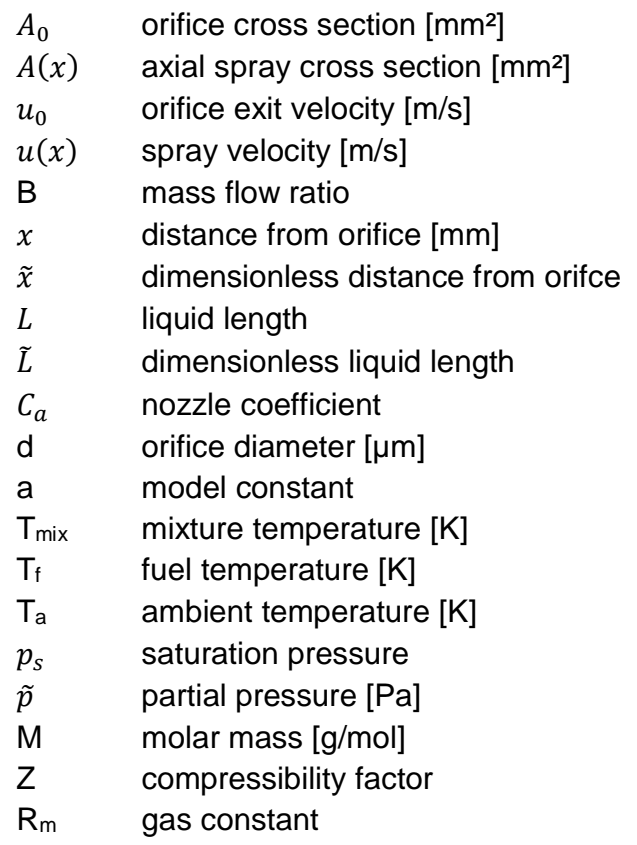

\section{References}

[1] Naber, J., and Siebers, D. L., 1996, "Effects of gas density and vaporization on penetration and dispersion of diesel sprays," No. 0148-7191, SAE technical paper.

[2] Siebers, D. L., 1999, "Scaling liquid-phase fuel penetration in diesel sprays based on mixing-limited vaporization," No. 0148-7191, SAE technical paper.

[3] Yue, Y., Powell, C. F., Poola, R., Wang, J., and Schaller, J. K., 2001, "Quantitative measurements of diesel fuel spray characteristics in the near-nozzle region using X-ray absorption," Atomization and sprays, 11(4).

[4] Leick, P., Riedel, T., Bittlinger, G., Powell, C., Kastengren, A., and Wang, J., "X-ray measurements of the mass distribution in the dense primary break-up region of the spray from a standard multi-hole common-rail diesel injection system," Proc. 21st Annual ILASS-Europe Conference.

[5] Riess, S., Klima, T., Wensing, M., and Braeuer, A., 2016, "Influence of Bio-Diesel and Ethanol on the Diesel Engine Process Chain Investigated by Optical Measurement Techniques," ILASS - Europe Brighton, UK.

[6] Wu, Z., Zhu, Z., and Huang, Z., 2006, "An experimental study on the spray structure of oxygenated fuel using laser-based visualization and particle image velocimetry," Fuel, 85(10-11), pp. 1458-1464.

[7] Linstrom, P. J., and Mallard, W. G., 2001, "The NIST Chemistry WebBook: NIST Standard Reference Database Number 69," Journal of Chemical \& Engineering Data, National Institute of Standards and Technology, Gaithersburg MD, 20899, http://webbook.nist.gov. 